\title{
Event memory and moving in a well-known environment
}

\author{
Andrea K. Tamplin • Sabine A. Krawietz • \\ Gabriel A. Radvansky • David E. Copeland
}

Published online: 12 June 2013

(C) Psychonomic Society, Inc. 2013

\begin{abstract}
Research in narrative comprehension has repeatedly shown that when people read about characters moving in well-known environments, the accessibility of object information follows a spatial gradient. That is, the accessibility of objects is best when they are in the same room as the protagonist, and it becomes worse the farther away they are see, e.g., Morrow, Greenspan, \& Bower, (Journal of Memory and Language, 26, 165-187, 1987). In the present study, we assessed this finding using an interactive environment in which we had people memorize a map and navigate a virtual simulation of the area. During navigation, people were probed with pairs of object names and indicated whether both objects were in the same room. In contrast to the narrative studies described above, several experiments showed no evidence of a clear spatial gradient. Instead, memory for objects in currently occupied locations (e.g., the location room) was more accessible, especially after a small delay, but no clear decline was evident in the accessibility of information in memory with increased distance. Also, memory for objects along the pathway of movement (i.e., rooms that a person only passed through) showed a transitory suppression effect that was present immediately after movement, but attenuated over time. These results were interpreted in light of the event horizon model of event cognition.
\end{abstract}

Keywords Event cognition $\cdot$ Mental models $\cdot$ Situation models $\cdot$ Updating $\cdot$ Space

\footnotetext{
A. K. Tamplin $(\bowtie) \cdot$ S. A. Krawietz $\cdot$ G. A. Radvansky Department of Psychology, University of Notre Dame, Notre Dame 46556, Indiana

e-mail: atamplin@nd.edu

D. E. Copeland

Department of Psychology, University of Nevada, Las Vegas, Nevada, USA
}

The structure of environmental space influences how we think about the world. Research using interactive environments has shown that the availability of knowledge about objects in an environment is affected by the structure of the environment itself (Radvansky \& Copeland, 2006c; Radvansky, Krawietz, \& Tamplin, 2011; Radvansky, Tamplin, \& Krawietz, 2010), because memory is directly impacted by a person's location in an environment, as well as by movement through it. The present research focused on the accessibility of knowledge as a person moves through a well-known environment. In other words, how does moving through a memorized space influence the accessibility of information about objects that are in different locations within that space?

\section{Spatial gradient of availability}

Situation model theory (Zwaan \& Radvansky, 1998) suggests that people create a representation that is continuously updated to coincide with the current state of affairs described by a text. Several important findings have been revealed in narrative comprehension research concerning how readers interpret and remember spatial information when they mentally track the location of the protagonist in a story. The finding of particular concern here is how the accessibility of environmental objects in memory (e.g., how quickly one can recognize two objects as being in the same room) changes as a protagonist moves through the environment (e.g., Bower \& Morrow, 1990). In these studies, a person first memorizes a map of an area, such as a building (see Fig. 1), along with the locations of objects within that environment. Then the person reads a story describing events within that environment.

In this research tradition, information accessibility is typically assessed after the story protagonist has made a movement from one location to another. The room from 
Fig. 1 Map of a research center used in these experiments.

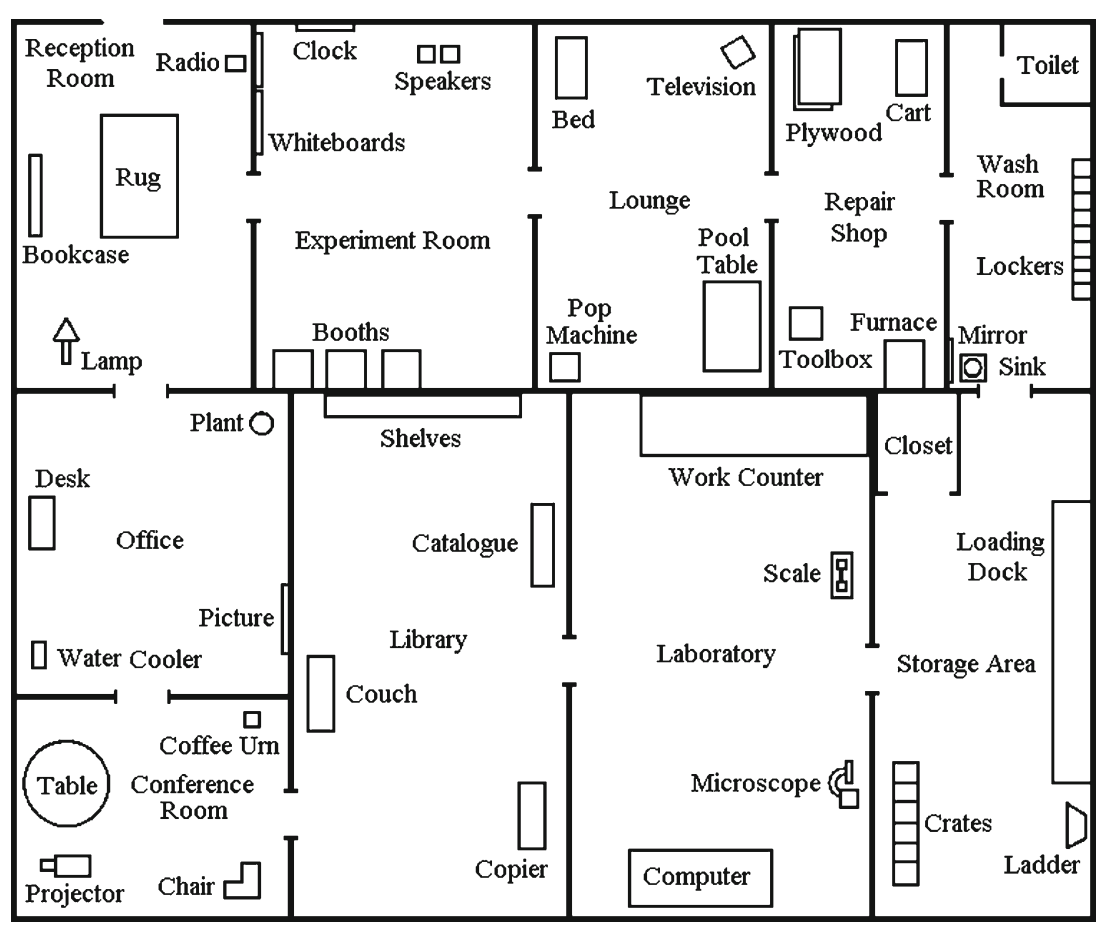

which the movement originated is called the source room, as it is the source of the movement. The room that the person passes through is called the path room, because it is along the path of travel. The room that the person currently occupies (i.e., after the movement) is called the location room, because it is where the person is currently located. Finally, any remaining rooms are referred to as other rooms and serve as a baseline from which to assess the accessibility of information about objects in the first three room types.

During reading, knowledge of objects in the building is probed for, either through explicit memory probes for pairs of objects (e.g., Morrow, Bower, \& Greenspan, 1989; Morrow, Greenspan, \& Bower, 1987; Rapp, Klug, \& Taylor, 2006; Stine-Morrow, Morrow, \& Leno, 2002) or by assessing reading times of critical sentences that refer to single objects in the building (e.g., Curiel \& Radvansky, 2002; Dutke, 2003; Dutke, Ribback, \& Wagner, 2003; Rinck \& Bower, 1995, 2000). As the story protagonist moves through the known space, objects in the location room (i.e., the protagonist's current location) are the most easily accessible. This accessibility is followed by objects in the path room, and then in the source room, during reading. Objects in the other rooms are least accessible, because they are the farthest away from the protagonist's current location. Note that the path room is never explicitly mentioned in the text during the movement, and response times for this condition are typically slower than those for the location room, and faster than those for the source room. This decrease in the availability as a function of the distance from the story protagonist's current location is the spatial gradient of availability.
The typical account for this finding uses a foregrounding explanation (Glenberg, Meyer, \& Lindem, 1987). When a story protagonist moves from one location to another, objects that are associated with him or her are foregrounded in working memory, making them more available. In comparison, entities that are dissociated from the protagonist are less available. As the protagonist moves, readers mentally update what is in the focus of their spatial map, and the farther objects are from the protagonist, the farther they are from the foreground of the situation model. Here, we refer to this traditional view of the spatial gradient of availability as a fading-foreground account.

This spatial gradient occurs regularly, even when the memory organization may be more temporal than spatial (Curiel \& Radvansky, 2002) or when the story protagonist is not actually moving, but is only thinking about traveling through the building (Rinck, Williams, Bower, \& Becker, 1996). Furthermore, this effect is governed by the number of rooms that are traversed rather than a reflection of Euclidean distance (Rinck \& Denis, 2004; Rinck, Hähnel, Bower, \& Glowalla, 1997). Importantly, the reader needs to mentally track the protagonist through the building. When people do not track the protagonist, such as when the name of the protagonist is not included among the pairs of probe items, the spatial gradient is not observed (Wilson, Rinck, McNamara, Bower, \& Morrow, 1993). This spatial gradient of availability is more prominent when the movement is in a continuous, expected direction, as compared to when the movement from room to room is more random (e.g., jumping from room to room via security cameras) (Dutke \& Rinck, 2006; Rapp et al., 2006). Finally, the reader needs to have 
extensive knowledge of the spatial layout prior to reading, through either memorizing a map or reading a spatial description of the environment that associates the objects with the rooms that they are in prior to reading the narratives (Haenggi, Kintsch, \& Gernsbacher, 1995; Rinck et al., 1996). Without such knowledge, the spatial gradient is not observed (Zwaan, Radvansky, Hilliard, \& Curiel, 1998).

\section{Moving through space}

One of the underlying ideas driving much of the research on narrative comprehension involving the spatial gradient effect is the idea that as people are reading, they are creating situation models that are, in essence, mental simulations of the story world, as if they themselves were travelling through that environment. This is supported by work showing that if people do not read narratives about story characters, but instead read instructions that encourage them to imagine themselves moving through the environment, a similar spatial gradient effect emerges (Rinck et al., 1996). Although such findings support the idea that narrative comprehension involves a mental simulation of the described narrative worlds, this does not mean that such imagined environments are faithfully experienced or are processed identically to a real, interactive environment.

Some principles derived from narrative event comprehension studies have been successfully extended to interactive environments (Radvansky \& Copeland, 2006c; Radvansky et al., 2011, 2010). These were conceptual replications of a study done by Glenberg et al. (1987; see also Radvansky \& Copeland, 2001, 2010). In a study by Radvansky and Copeland (2006c), people navigated a desktop virtual environment, picking up and setting down objects. When people moved from one room to another, there was a decline in memory for objects that were interacted with. That is, moving through doorways, as opposed to moving an equal distance in the same room, caused forgetting. This finding suggests that human cognition can be meaningfully affected by the structure of the environment and how a person interacts with it. In the present research we further explored this phenomenon. It should be noted that because the term situation model is so strongly associated with research in narrative comprehension, we use the more general term event model here (e.g., Kurby \& Zacks, 2008; Radvansky \& Tamplin, 2012; Radvansky \& Zacks 2011; Swallow, Zacks, \& Abrams, 2009).

The present research is interpreted in the context of the event horizon model of event cognition (Radvansky, 2012; Radvansky \& Tamplin, 2012; Radvansky \& Zacks, 2011). This view assumes that retrieval from event models is influenced by five principles: (a) First, experience is segmented into event models that are stored separately in memory; (b) second, the current event model is more highly available; (c) third, the causal connections among events are encoded and influence retrieval; (d) fourth, noncompetitive attribute retrieval occurs when information is distributed across multiple events; and (e) fifth, competitive event retrieval occurs when retrieval accesses multiple event models as part of the retrieval process (see Radvansky, 2012; Radvansky \& Tamplin, 2012; Radvansky \& Zacks 2011, for further details). The present project involves the first, second, third, and fifth principles.

When people experience events, they break the information down into different event segments (e.g., Swallow et al., 2009). A location boundary is a specific type of event boundary that occurs when there is a change in location, such as moving from one room to another. This feature is one of the several kinds of event boundaries that are identified by most people. The consequence of this segmentation is that at each location boundary, people update their event model to capture the new event information. Moreover, when information is currently being processed, information from the current event model is more available than is other event information. Thus, as a consequence of this shift, the prior model declines in availability.

Research on narrative comprehension has shown that objects farther away are less available. If interactive experience is similar, then a spatial gradient will be observed. However, reading is qualitatively different from an interactive experience. For example, knowledge of one's current location is perceptually available, and locations that are not mentioned in a text, such as a path room, are actively experienced as part of a continuous movement. This may influence the availability of information from that room.

\section{Event horizon model account}

Now that we have identified the experimental paradigm for the present study and outlined the event horizon model, we will look at the predictions that it makes in comparison to the traditional fading-foreground account of the spatial gradient effect that has been applied to narrative comprehension contexts. The predictions of the event horizon model are shown in Fig. 2. First, consider the text comprehension paradigm (Fig. 2A). Initially, people memorize a map of the building and the objects in the various rooms. Consistent with a wealth of research on mental maps (e.g., Carbon \& Leder, 2005; Curiel \& Radvansky, 1998; Friedman \& Brown, 2000; McNamara, 1986; Stevens \& Coupe, 1978; Thorndyke, 1981), this is likely to be divided into regions based on the rooms in the building. When people read the narratives that take place in this setting, they track where a story protagonist is by referring to this mental map. This is done by activating the rooms, one at a time, to move through the chunks (subregions or rooms). 
Fig. 2 Predictions of the event horizon model, based on whether a person is $(\mathbf{A})$ reading a narrative text or $(\mathbf{B}, \mathbf{C})$ moving within an interactive environment: (B) without or (C) with a path room. a

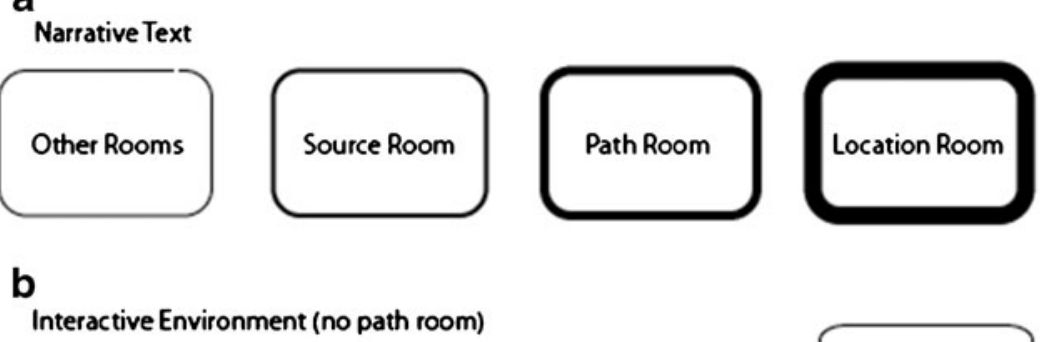

Interactive Environment (no path room)
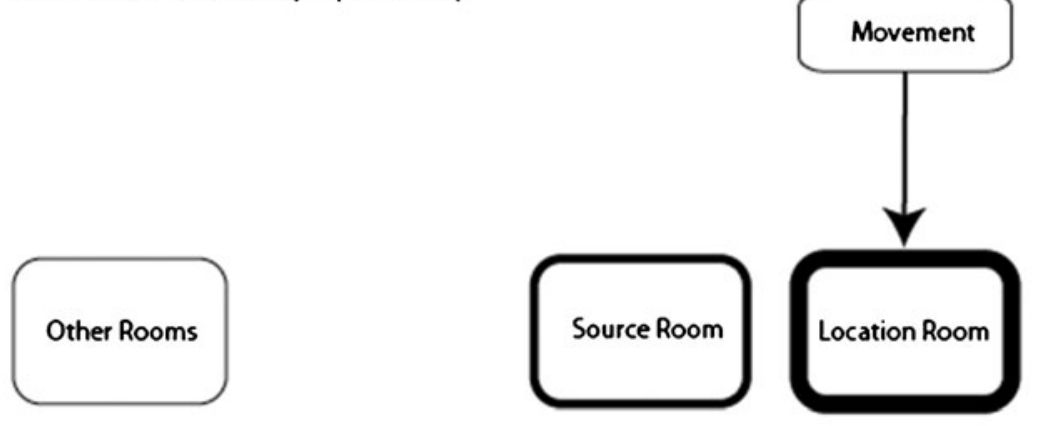

C

Interactive Environment (with path room)

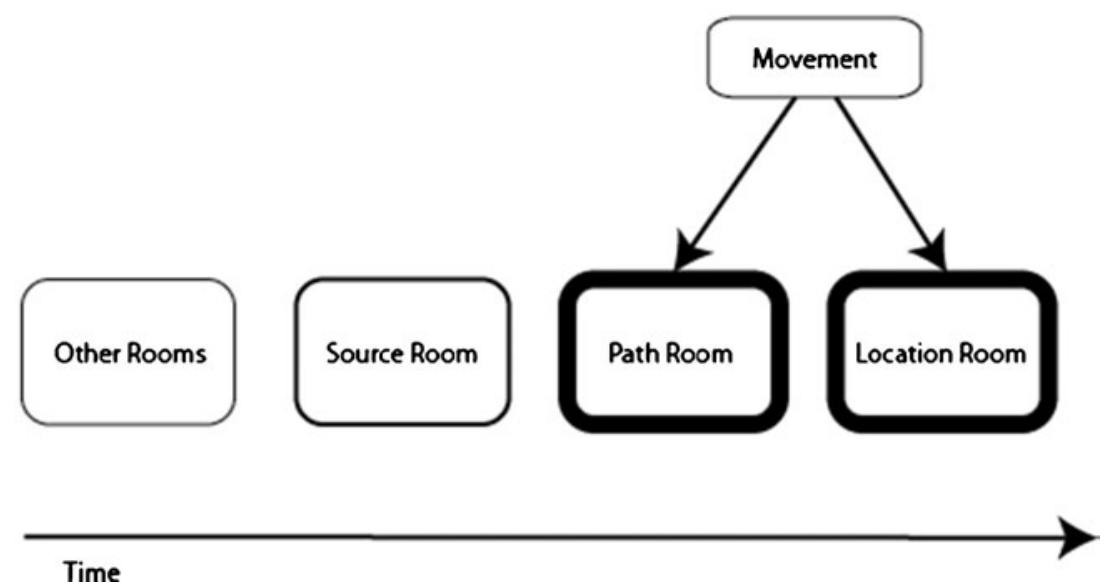

For the event horizon model, the critical parts while reading are event segmentation and the higher availability of the current event model. Thus, each room that a protagonist enters, via segmentation, results in a new event model. As a character moves through the building, the creation, activation, and decay of event models occur in a room-byroom fashion, with the current location room being the most available, and the other rooms declining in availability as a function of how long it has been since they were activated. As a result, a standard spatial gradient effect emerges. This is consistent with the traditional fading-foreground account of the spatial gradient of availability.

Now consider the predictions for interactive environments, as studied here. After map memorization, people would have mental maps similar to those in the narrative studies. However, people are less dependent on that mental map because they can see the rooms that they are in. For the event horizon model, as with narratives, when people are currently in a room, the event model for that room is most activated. However, with interactive events, movement is a continuous flow through the environment. When moving from one room to the next (see Fig. 2B), if there is no path room, the occupations of the source room and the location room are separate events, and so are captured by two event models. The event model for the new room is constructed and activated, and the model for the previous room begins to decay.

Now, consider when a path room is present (Fig. 2C). First, as per the first component of the event horizon model, in terms of parsing the stream of action, the source room is one event. This is the current event model that a person is processing when the instruction to move is received. In comparison to this, the movement through the path room into the location room is a continuous process and is a separate, single course of events (Barwise \& Perry, 1983). That is, the occupation of each room can be a separate event, 
but these events are joined by two types of causal information (the third component of the event horizon model): information (a) that the goal of the movement is to arrive in the location room and (b) that the path room must be crossed to reach the location room. Thus, the movement involves a superordinate movement structure that includes the path and location rooms.

With regard to the second component of the event horizon model, during movement, the event model for the source room ceases to be the current model and begins to fade. Within the context of the larger causal sequence of events that composed the movement are two event models, one for the path and one for the location rooms, and these are joined by the causal sequence that makes up the movement. During this movement, the path room is a salient part of the movement, in that a person actually experiences it perceptually, along with some retrieval of the information associated with that room (the objects). However, the event model for that room is not the goal of the movement, the location room is. As such, the path room is salient, but irrelevant. The event model for the path room is an active source of interference relative to accessing knowledge of the goal of that movement, the location room. That is, because both rooms are part of the same movement, accessing information from either one of these event models will set up a competition between the two. The presence of two mental representations (the event models) that share a concept (the movement) can be thought of as a form of associative interference and a type of fan effect, because the two locations compete for retrieval from long-term memory as a consequence of being associated with the same retrieval cue (the movement; Radvansky, 1998, 1999a, 2005, 2009; Radvansky \& Copeland, 2006a, 2006b; Radvansky, Spieler, \& Zacks, 1993; Radvansky \& Zacks 1991). This is where the fifth component of the event horizon model comes in. Thus, although there is some facilitation of the location room because it is the current event model, there is also interference during retrieval because the path and location rooms compete with one another.

\section{Overview of experiments}

The aim of the present study was to examine whether the spatial gradient found in text comprehension extends to an interactive environment. We assessed this in Experiments 1, 2 , and 3 . The primary differences between these experiments were the range of locations probed for and the timing of the probes. Experiment 1 was modeled after a study by Morrow et al. (1987), in which people were probed about information in the location and source rooms, but a path room was not included. For Experiment 2, the same procedure was used, except that a path room was now included. In
Experiment 3, a 2-s delay was added before the probe to allow for event model updating to complete.

In all of these experiments, people memorized the map of a research center using the same procedure as had people in previous narrative comprehension studies (e.g., Curiel \& Radvansky, 2002). Thus, after memorization, people had the same knowledge as people in narrative comprehension studies. However, what differed was how they experienced the memorized environment, and, hence, how the memorized knowledge was used. In the present experiments, people navigated a virtual environment.

\section{Experiment 1}

Experiment 1 was modeled after a text comprehension study by Morrow et al. (1987), which included location and source rooms that were adjacent to each other; no path rooms were used. The major difference was that instead of reading a narrative, people navigated a virtual environment. As in the narrative studies, people were probed with pairs of objects and indicated whether the two objects were in the same room. Again, the traditional fading-foreground account of the spatial gradient effect predicts that information in the location room will be most available, followed by information for the source room and the other room. The event horizon model makes a similar prediction. The importance of this experiment is that, whereas the event horizon model predicts interference from a path room when one is present, it is not the case that any room just prior to the location room will be a source of interference. Specifically, when the prior room is the source room, no interference is predicted, because that room is not part of the goal. Thus, the aim of Experiment 1 was to demonstrate that the room just prior to the location room would not be as disrupted, as would be the case for the path rooms in the following experiments.

\section{Method}

Participants A group of 28 undergraduates were recruited from the University of Notre Dame and received partial class credit for their participation.

Materials and procedure A map of a research center, used by Curiel and Radvansky (2002), with ten rooms and four objects in each room, was presented for memorization (see Fig. 1). During memorization, people viewed the complete map on a computer until they felt ready to be tested on the room names and object locations. After studying, a blank map was displayed with red squares in place of the objects. People typed the name of the object located at each particular square. This procedure was done for all of the object locations. After this test period, 
the original map was displayed again for studying. This study-test procedure continued until all of the objects and their locations had been correctly recalled twice (i.e., two perfect test scores).

After participants had learned all of the objects and locations in the map, they continued to the second phase. A three-dimensional first-person perspective model of the research center was displayed on a 66-in. rear projection Smartboard using a PC-compatible computer. The virtual building was created using the Valve Hammer environment creation program. This is the program used to create environments for the Half-Life video game. A sample display is shown in Fig. 3. People were seated about $1 \mathrm{~m}$ in front of the large display in order to increase the amount of their field of view that was filled with the virtual world. In addition, the testing room was darkened to minimize distractions and people wore headphones so that they could hear "their own footsteps" as they traveled.

People first traveled through all of the building rooms to familiarize themselves with the area, starting with the reception room. They navigated the virtual environment with their left hand by using the up, down, left, and right arrow keys to move forward, backward, left, and right, respectively. Traveling through the building was done from a firstperson perspective (i.e., not an overhead or birds-eye perspective), although no parts of the participant were visible on the screen. When people had returned to the reception room, the experimental trials began. Instructions appeared in the middle of the screen to direct the participant to move to another room (i.e., the location room) using the shortest path (as a reminder, in Experiment 1, this was an adjoining room for the critical trials). After arriving in the location room, they were given instructions to move to another room. This pattern of instructions continued until the end of testing. To make sure that people progressed through the rooms in the required order, in this and all of the experiments, a visual warning appeared on the screen if they chose the long route to the location room, indicating that the person was traveling in the wrong direction (i.e., "Wrong Way!").

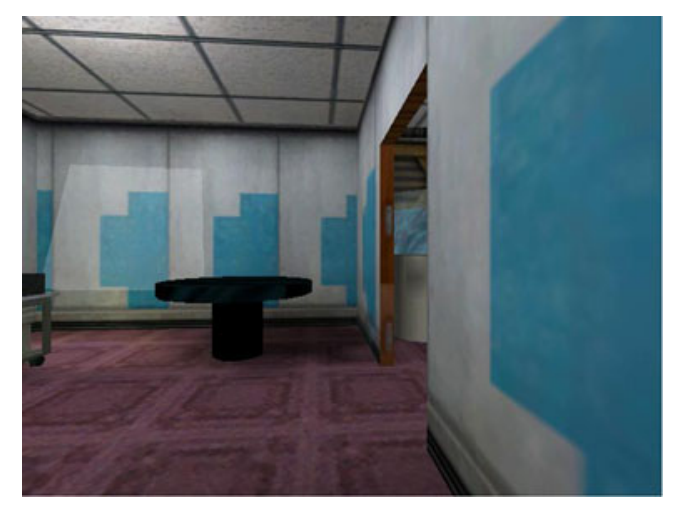

Fig. 3 View of one of the rooms in the virtual reality environment.
On critical trials, just after a person entered a new room, movement in the environment was stopped and a pair of object names were presented on the screen. On these trials, the image of the room that a person was in dimmed and the names of the probe objects appeared on the screen. Moreover, people could not move or turn about when the probes were on the screen. Note that when one or both of the objects was in the current room, these were objects that would be out of the person's view based on the trajectory with which the person entered the room. Thus, people were not responding to an object that was in their field of view. The task was to indicate whether the two objects occupied the same room in the building. Whereas positive trials featured objects in the same room, negative trials featured two objects that were in different rooms. For one out of every eight trials, the word "Yourself" was substituted for one of the two objects, and people were told to answer "Yes" if at that time they were in the room with that particular object. The probe appeared in the middle of the screen. Not every movement (i.e., instruction to move to another room) was accompanied by a memory probe; the probes occurred at various times, ranging from every zero to three moves. The negative probes were generated by combining two objects that were in different rooms. "Yes" and "No" responses were made by pressing one of two buttons on a computer mouse (the left and right buttons, which were labeled with a "Y" and "N," respectively). Because only the left hand was used to navigate the environment, the right hand was free to remain on the mouse in order to assure immediate responding. The experimental procedure typically lasted $1 \mathrm{~h}$.

For Experiment 1, the probes were for the location, source, and other rooms, with the location and source rooms being adjacent. Eight positive probes were presented per condition (24 total). The positive probes were counterbalanced with 24 negative probes. There was no feedback; after participants had responded, they continued to move about in the environment by following the next movement instruction. In Experiment 1, the probes were presented right after entering the target room (after about one step into the room).

\section{Results and discussion}

The response time and error rate data for Experiment 1 are presented in Table 1. All room comparisons were made to explore the nature of the availability of information. Only the response times for correct trials were considered; also, those that were either greater than $10,000 \mathrm{~ms}$ or less than $500 \mathrm{~ms}$ were trimmed as being unreasonably slow or fast, followed by a standard deviation trim based on the individual sample size (see Van Selst \& Jolicœur, 1994). This adjustment resulted in a loss of $1.3 \%$ of the response time data. Also note that 
Table 1 Mean response times (in milliseconds) and error rates (as proportions) for the first set of interactive environments in Experiments 1,2 and 3

\begin{tabular}{lllll}
\hline & Location & Path & Source & Other \\
\hline Response Times & & & & \\
Experiment 1 & $2,266(130)$ & & $2,247(134)$ & $2,473(135)$ \\
Experiment 2 & $2,198(46)$ & $2,475(60)$ & $2,312(55)$ & $2,302(51)$ \\
Experiment 3 & $2,130(75)$ & $2,355(67)$ & $2,468(93)$ & $2,378(81)$ \\
Error Rates & & & & \\
Experiment 1 & $.05(.02)$ & & $.07(.02)$ & $.09(.02)$ \\
Experiment 2 & $.07(.01)$ & $.09(.01)$ & $.08(.01)$ & $.06(.01)$ \\
Experiment 3 & $.04(.01)$ & $.09(.01)$ & $.09(.01)$ & $.05(.01)$ \\
\hline
\end{tabular}

Standard errors are presented in parentheses.

on no trials had the source room been the location room on the previous trial, so as to avoid trials that could produce a repetition priming effect. This would happen because people would be probed about a room in which they had just received a probe.

For Experiment 1, responses in both the location and source room conditions were faster than those in the otherroom condition, $F(1,27)=4.23, M S E=142,335, p=.05$, and $F(1,27)=8.82, M S E=81,038, p=.006$, respectively, and these two conditions were not significantly different from one another, $F<1$. Thus, responses to the most recently occupied and current locations were faster than those to any other room in the building.

The error rate data were submitted to similar analyses. We found a significant benefit for the location room relative to the other rooms, $F(1,27)=5.87, M S E=.005, p<.05$, but not between the source and other rooms, $F<1$, or the location and source rooms, $F(1,27)=1.22, M S E=.006, p>.05$. Thus, some evidence of facilitated processing emerged for the location room, and the source room's results were between that level and the other-room baseline.

These results suggest that the availability of information in memory during active interaction only partially parallels the data observed in research on language comprehension. Specifically, consistent with previous research showing a spatial gradient, people were faster to respond to probes for objects in either the currently or recently occupied locations. Moreover, people were most accurate for objects in the location room, least accurate for objects in the otherroom condition, and objects from the source room occupied the region between these two. However, inconsistent with the fading-foreground view, we found no response time difference between the location and source room conditions. This finding may reflect the facts that the source room had just been occupied and that the activation level had not had enough time to decay. This idea was explored further in Experiments 2 and 3.

\section{Experiment 2}

The aim of Experiment 2 was to assess whether a spatial gradient would be observed with interactive environments when a path room was included. The majority of studies in narrative comprehension have included such rooms, and their inclusion allows for a wider range of rooms in which to potentially observe a spatial gradient, if it exists. In the fading-foreground view, a spatial gradient should be observed: Objects in the location room should be most accessible, followed by those in the path room and then the source room. The event horizon model predicts that the path room information will show an effect of suppression, because it is a source of interference competing with the activation of the location room, as they are both part of the current event (i.e., the movement), but the path room is irrelevant to the goal (i.e., arriving at the location room). Finally, the event horizon model also predicts that because more time will pass between leaving the source room and the arrival in the location room, the accessibility of the source room should decay relative to what was observed in Experiment 1.

\section{Method}

Participants A group of 180 undergraduates were recruited from the University of Notre Dame and received partial class credit for their participation.

Materials and procedure The materials and procedure were similar to those of Experiment 1 (i.e., memorization, keypresses, warnings about moving in the wrong direction, etc.), except that a path room was now present on probe trials. That is, prior to a critical probe, people had to travel from the source room, through the path room, and to the location room prior to getting an object pair probe. Again, these probes were presented in random order, and for negative probes, people were given the names of objects from two different rooms. Six positive probes were presented per condition (i.e., in source, path, location, and other rooms: 24 total), and the positive probes were counterbalanced with 24 negative probes.

\section{Results and discussion}

The response time and error rate data for Experiment 2 are presented in Table 1 . The data trimming procedure resulted in a loss of $6 \%$ of the response time data.

For Experiment 2, responses were slower for the path room probes than for the location, source, and other-room conditions, $F(1,179)=30.02, M S E=228,990, p<.001 ; F(1,179)=$ 9.60, $M S E=194,677, p=.002$; and $F(1,179)=12.29, M S E=$ $217,251, p=.001$, respectively. Moreover, responses for the 
source room probes were slower than those for the location room probes, $F(1,179)=5.62, M S E=207,417, p=.02$, but not than for the other-room probes, $F<1$. This suggests that the availability of the information in the source room was back at baseline. This differed from the results of Experiment 1 because, in Experiment 2, the presence of the path room caused enough time to pass after leaving the source room for its accessibility to decay. Finally, the location room probes were responded to faster than the other-room probes, $F(1,179)=6.73, M S E=144,939$, $p=.01$, consistent with the idea that the currently occupied location is part of the current event model.

Thus, when a path room was introduced, responses to this condition were slower than those to the location and otherroom conditions. This is consistent with the idea that the path room, which was passed through but was not the goal of travel, was a source of interference because it was highly salient and perceptually available but was irrelevant to the aim of travel. Finally, the faster responses to the location room suggest that information that was in this currently occupied location had becoming activated.

As for the error rate data, although the pattern of errors nominally mirrored that of the response time data, none of the differences were significant, all $p \mathrm{~s}>.10$.

These results deviated from the classic spatial gradient effect that is observed in research on language comprehension. Although performance was best in the location condition, performance was worst in the path room condition and was in between for the source and other-room conditions. Using the other-room condition as a baseline, it appears that information in the location room was facilitated because it was part of the current event model. Information in the source room was back at the baseline level of availability, which differs from the results from research on language comprehension. Most interestingly, we found a response time suppression effect for responses to the path room probes.

One possible explanation for this pattern is that it is strictly the result of an interference effect. That is, the increased availability of objects in the location room interfered with the ability to access information in the path room, which was most adjacent to the location room. Because information in the location room was at a higher level of activation, this outshined and competed with the ability to access highly related information, such as that in the path room.

Alternatively, an inhibition account would argue that the path room information was salient because people were passing through it as they tried to complete their goal. Moreover, it was also irrelevant because people were not planning on stopping in that room. A typical fate for such strong, but wrong, information is that it is inhibited in memory (e.g., Hasher \& Zacks, 1988; Radvansky, 1999b).
In other words, a consequence of the interference effects of such salient but irrelevant information is that cognition would actively inhibit them, to keep them from intruding on the current stream of processing.

\section{Experiment 3}

The aim of Experiment 3 was to test the interference and inhibition accounts of the path room suppression effect observed in Experiment 2. To do this, in Experiment 3 a 2-s delay was added prior to the presentation of a memory probe (i.e., the memory probe occurred $2 \mathrm{~s}$ after a person entered the location room, instead of immediately). The choice of a 2-s delay was somewhat arbitrary, but it was thought to be long enough for event model updating to progress or to complete after the person had entered the location room. Part of the motivation for this choice was that, in Experiment 2 relative to Experiment 1, $2 \mathrm{~s}$ was the approximate amount of time added to a trial by including the path room, and when adding this time, the source room activation level had decreased to baseline levels in Experiment 2. On the basis of this reasoning, we determined that $2 \mathrm{~s}$ would likely be a sufficient amount of time for a decay process to make meaningful progress toward event model updating. If the path room response time slowdown were due to interference, the continued activation of the location room information should continue to disrupt access to the path room, and a significant slowdown would be observed. In contrast, if this effect were due to inhibition, a 2-s delay would allow the activation level of the path room to dissipate, reducing it as a source on interference, and removing the need for it to be actively inhibited. As in Experiment 2, the fading-foreground view would again predict a typical spatial gradient effect.

\section{Method}

Participants A group of 80 undergraduates were recruited from the University of Notre Dame and received partial class credit for their participation.

Materials and procedure The same materials and procedure were used as in Experiment 2, except that after a location room was entered, movement was frozen for a 2-s delay before the probes were presented. Besides that, the materials and procedure were identical to those of Experiment 2.

\section{Results and discussion}

The response time and error rate data are presented in Table 1. The data trimming resulted in a loss of $6 \%$ of the response time data. People responded faster to the location 
room probes than to the path, source, and other-room conditions, $F(1,79)=9.81, M S E=205,976, p=.002$; $F(1,79)=10.71, M S E=426,496, p=.002$; and $F(1,79)=6.61, M S E=373,374, p=.01$, respectively. The remaining three conditions did not differ from one another, all $p \mathrm{~s}<.25$. Thus, we observed facilitation for information in the person's current location but no difference in the availability of information from the other locations, outside of the current event model. Moreover, the path room suppression effect seen in Experiment 2 was no longer evident. This result is more consistent with a transitory inhibition account than with interference.

For the error rate data, the location condition was significantly more accurate than the path and source room conditions, $F(1,79)=10.77, M S E=.009, p=.002$, and $F(1,79)=$ $14.36, M S E=.006, p<.001$, respectively, but not than the other-room condition, $F<1$. Moreover, the path and source conditions did not differ from one another, $F<1$, but both of these conditions had more errors than the other-room condition, $F(1,79)=5.71, M S E=.010, p=.02$, and $F(1,79)=5.78$, $M S E=.009, p<.02$, respectively. This error rate pattern may reflect some residual processing difficulty, because the path and source rooms had recently been occupied. However, given that this sort of error rate pattern did not emerge in either Experiment 1 or 2, any interpretation of these error rates would be quite speculative.

In contrast to narrative comprehension research, the results of Experiment 3 did not show evidence of the traditional spatial gradient effect. Instead, event model updating in an interactive environment was largely confined to the activation of a person's current location, and we did not find a graded pattern of availability with increased distance. In addition, a transitory suppression of information from the path room dissipated as more time elapsed since the person had last occupied that room. Whereas this is inconsistent with an account based on interference by the current activation of the location room on the path room, it is consistent with an account based on the inhibition of the path room when it is highly salient but irrelevant.

\section{General discussion}

The results of the research reported here, involving interactive environments, differ from the pattern of data observed with narrative texts. The present study showed some evidence of facilitated processing for information in the location room, but the patterns for the other conditions did not show the standard pattern. Importantly, we saw no clear spatial gradient effect, as is typically observed in narrative memory (Bower \& Rinck, 2001; Curiel \& Radvansky, 2002; Dutke, 2003; Dutke et al., 2003; Dutke \& Rinck, 2006;
Morrow et al., 1989, 1987; Rapp et al., 2006; Rinck \& Bower, 1995; Rinck, Bower, \& Wolf, 1998; Rinck et al., 1997). This is inconsistent with the more traditional fadingforeground account. It is important to keep in mind that our claim is not that people do not mentally simulate narrative worlds, but that such mental simulation, while capturing some aspects of actual experience, differs in meaningful ways from it.

The increased availability of information in the location room is consistent with the idea that people segment the ongoing stream of experience into events, and that these event boundaries occur when a person moves from one room to another. Information that is part of the current event model is at a higher degree of activation, seen in the increased availability of information for the location room. This pattern was expected because, despite the differences between the virtual environment and those of narrative-based studies, the current location was a relevant part of the current movement goal, and this is true for both tasks.

We also observed a suppression effect for the path room, although this was transient. It was present when people had just moved through a path room, but dissipated when the probe was delayed by $2 \mathrm{~s}$. This is consistent with the idea that the information in the path room was salient as a person walked through it, producing interference, and hence, suppression of that source of interference. However, because the activation of the path room did not continue, the need to inhibit it was lessened over time, and the suppression effect dissipated. This pattern of data is inconsistent with an interference account, in which the activation of the location room information interferes with and blocks access to information from the path room. If this were the case, as the facilitation of the location room continued across the 2-s delay, there should have been continued interference with the path room information; however, this did not occur.

Overall, the results of the present study cannot be explained by a traditional fading-foreground account of the spatial gradient. However, the event horizon model can account for the observed patterns of data in both the narrative comprehension and interactive environment studies. First, for narrative comprehension studies, as noted earlier, the standard fading-foreground account of the spatial gradient effect is due to people using mental maps, divided into regions based on the rooms. As people read, they track the story protagonist by referring to the mental map, activating the rooms one at a time with the movement. For the event horizon model, using the segmentation of the rooms into event models and the more activated status of the current event model, each of the rooms that a character goes into serves as a new event model. This movement results in the creation, activation, and decay of these models, with the current room being the most available, and the others declining in availability as they become further removed from the focus. 
Fig. 4 Comparison of human and predicted data for narrative comprehension studies in which a reader is interrupted and probed with object name pairs.
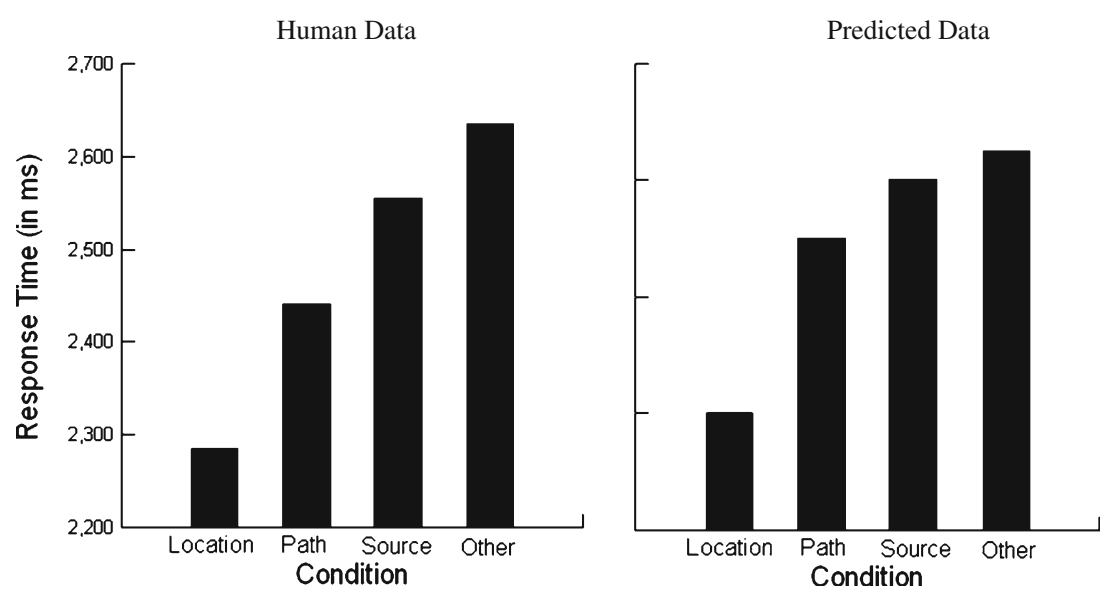

To better capture the processes suggested here, we simulated the retrieval of information from the various room-based event models using the principles embodied in the hydrogen model of memory retrieval (see Radvansky \& Tamplin, 2012). This model essentially assumes a division of activation based on the number of mental representations involved during retrieval, as well as an allocation of active inhibition based on the amount of interference being experienced, which is similarly divided on the basis of the number of mental representations involved. Moreover, this model assumes a negatively accelerating function over time that brings representations back to a baseline level of activation.

Using this process, the simulated study for a narrative study is shown in Fig. 4. This account is compared with the pattern of data derived from actual experiments. For the human data, only two experiments have been reported in which (a) people memorized a map, (b) memory was assessed using probes, and (c) a path room was included. These experiments were Morrow, Bower, and Greenspan (1989, Exp. 1) and Wilson, Rinck, McNamara, Bower, and Morrow (1993, Exp. 4). Figure 4 presents the human and predicted data. The human data are weighted averages (based on the number of participants) of the data reported in the literature. As can be seen, similar spatial gradients occur in both the human and simulation data.
Now consider the interactive studies reported here. As in narrative studies, after map memorization, people would have a mental map to use. However, unlike a narrative, people are less dependent on it because they can see the rooms that they are in. Again, for the event horizon model, when a person is in a room, the current event model for it is most activated. When moving from that room to the next with no path room, as in Experiment 1, each room is a separate event. The two rooms, as different events, involve two event models. The one for the new room is constructed and activated, and the one for the previous room begins to decay. An important point for Experiment 1 is that the probe is presented immediately after a person has passed through the doorway. As such, there may not have been enough time for a new event model to be completely constructed, nor was there much time for the previous event model to decay. This may be why the two models are similarly available. As can be seen in Fig. 5, for both the human and predicted data, an advantage emerges for both the location and source rooms relative to the other-room probes.

Now consider the case in which a path room is present (Exp. 2). As we noted in the introduction, the movement through the path room into the location room is a continuous process and a single course of events (Barwise \& Perry,
Fig. 5 Comparison of human and predicted data for a virtual movement study (Exp. 1) in which there is no path room.
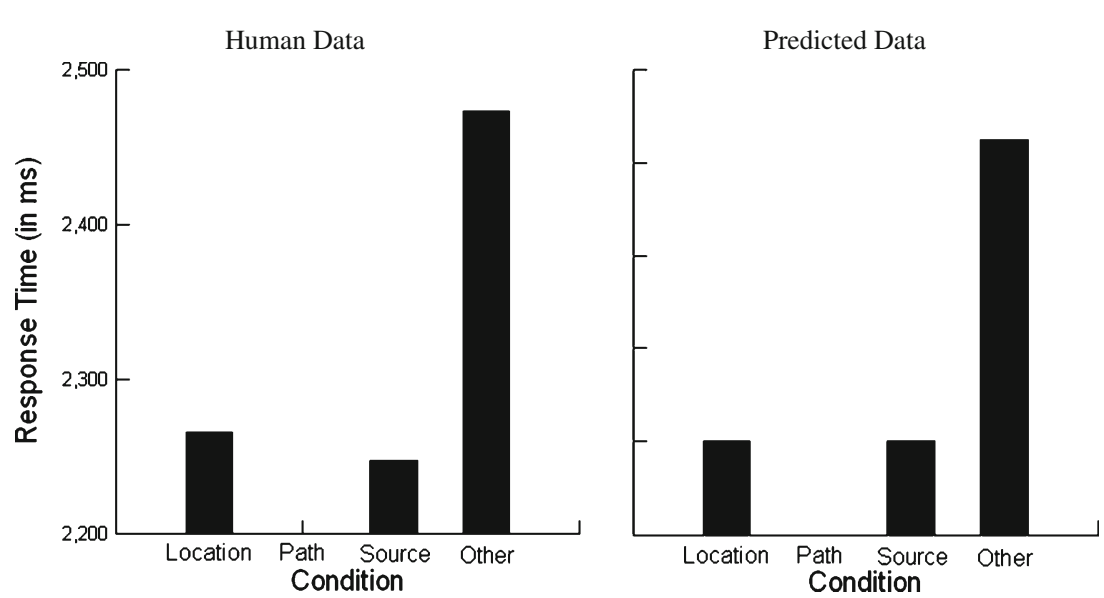
Fig. 6 Comparison of human and predicted data for a virtual movement study (Exp. 2) in which there is a path room.
Human Data

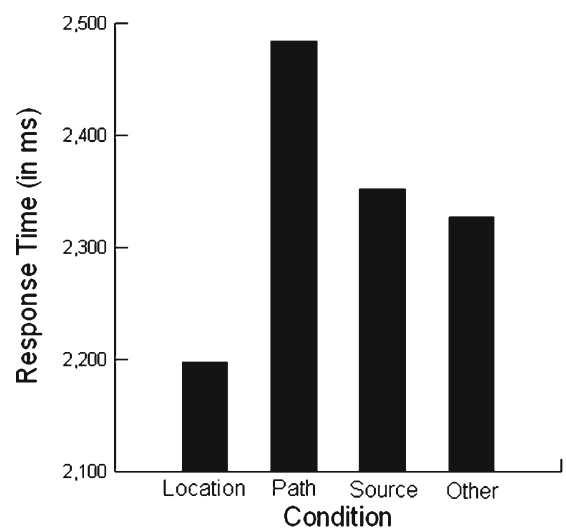

Predicted Data

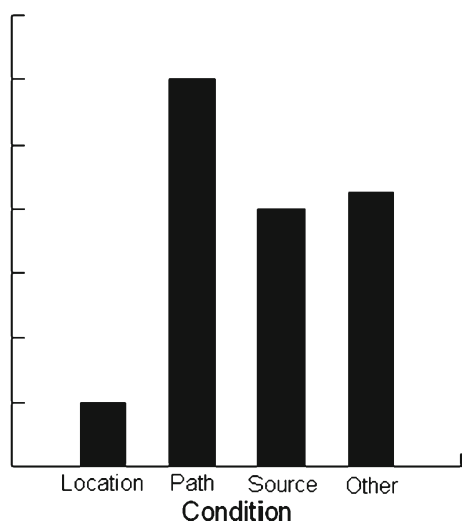

1983). Thus, the path room is a salient but irrelevant part of the movement, and it ultimately serves as a source of interference while the participant tries to accomplish the movement goal. This is where the fifth component of the event horizon model comes in. The path room representation, because it is related but irrelevant, according to our simulation, is suppressed in order to reduce this interference and aids the retrieval of information from the location room. Moreover, the source room is not suppressed because it is not part of the movement, and anyway, it has largely decayed back to baseline levels of availability at this point. The human data from Experiment 2 are presented in Fig. 6. The account provided by the event horizon model predicts a pattern of data similar to the one we observed.

Finally, consider the pattern of data when there is a path room and a delay before the probe (Exp. 3). Although there is interference, and thus suppression, the movement is now over, and the person is only focusing on the location room. At this point, the location room is more available, and because the path room is no longer interfering, it is no longer suppressed. The availability of information from this room returns to a baseline state, such as that observed with the other rooms. Finally, the additional delay has little to no effect on the source room, other than allowing it to decay to a baseline level. Thus, the predicted pattern of data is for facilitation of the location room, whereas the results for the other three room types are similar. The human data in Fig. 7 are from Experiment 3. As can be seen, the predicted pattern of data resembles the observed data.

Overall, the pattern of data is clearly more consistent with the event horizon model than with the traditional fadingforeground account of the spatial gradient effect. As aspects of an experience change, such as moving from one room to another, people segment their experience into event models. The current event model is most activated. Experiencing an event is different from reading about one, and results in the irrelevant information being more salient than it would be otherwise, producing the path room suppression effect. Also, the fact that a person was an active participant in the perceptually available event reduced the need to refer to a mental map to track spatial location, as is the case in narrative comprehension. The need to refer to mental maps likely produces the spatial gradient observed in those studies, whereas the absence of this demand eliminates the spatial gradient in an interactive environment.

\section{Conclusions}

The present findings extend studies of event cognition in order to provide a better understanding of the processes
Fig. 7 Comparison of human and predicted data for a virtual movement study (Exp. 3) in which there is a path room as well as a delay prior to the probe.
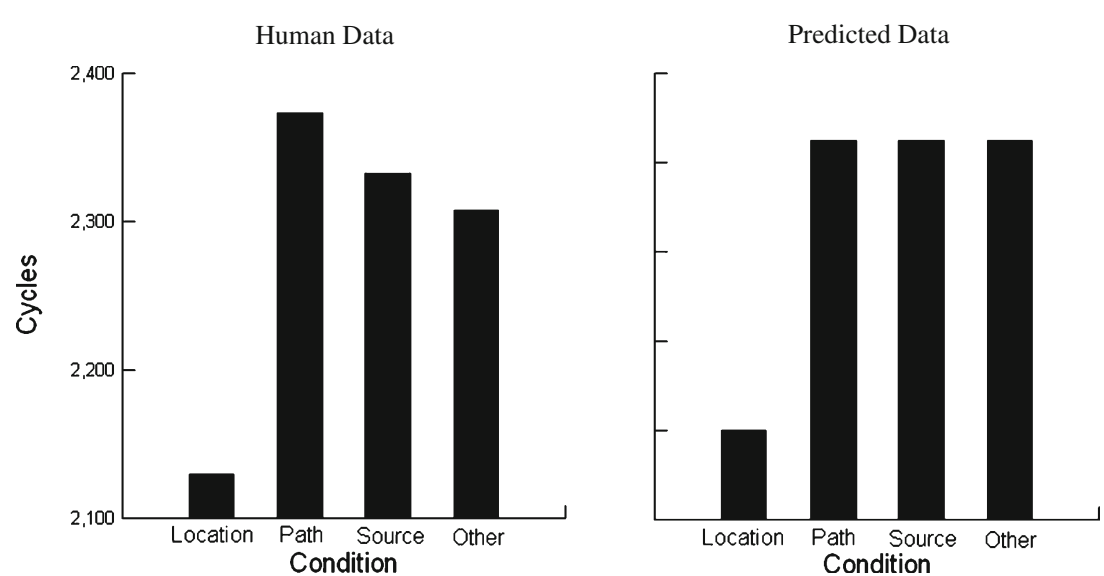
involved in location updating, and how this can vary under different circumstances. Moving through space, and the need to update one's event understanding, has influences on the ability to retrieve information. When this updating occurs during narrative comprehension, the availability of memories is influenced by the distance of items on a previously memorized map. However, in interactive events, the pattern of results is more complex and interesting: Whereas the current location is facilitated, perceptually salient but irrelevant information that was part of a travel path but was not the source or goal of that travel is, at least temporarily, suppressed. Moreover, spatial distance along a map or route does not guide the availability of information. Instead, availability is more a function of whether the information is part of the current environment/event.

Author note This research was supported in part by the Army Research Institute, Grant No. ARMY-DASW01-02-K-0003, and by funding from J. Chris Forsythe of Sandia National Laboratories. We thank Daniel Blakely, Chad Breeden, Megan Cefferillo, Davide Dal Grande, Abbi Daugherty, Jessica Harrison, Brittany Love, Tiffany Milligan, Patrick O'Keefe, Jenny Rinehart, Maureen Ritchey, Francie Schmuhl, Brian Stouffer, Chris Tulisiak, and Dan Wolters for their assistance in collecting the data. We also thank Jeff Smith for his programming expertise. Portions of this research were presented at the annual meeting of the Psychonomic Society in Houston, November 19, 2006.

\section{References}

Barwise, K. J., \& Perry, J. (1983) Situations and attitudes. Cambridge, MA: MIT Press.

Bower, G. H., \& Morrow, D. G. (1990). Mental models in narrative comprehension. Science, 247, 44-48. doi:10.1126/science.2403694

Bower, G. H., \& Rinck, M. (2001). Selecting one among many referents in spatial situation models. Journal of Experimental Psychology: Learning, Memory, and Cognition, 27, 81-98.

Carbon, C.-C., \& Leder, H. (2005). The Wall inside the brain: Overestimation of distances crossing the former Iron Curtain. Psychonomic Bulletin \& Review, 12, 746-750. doi:10.3758/ BF03196767

Curiel, J. M., \& Radvansky, G. A. (1998). Mental organization in maps. Journal of Experimental Psychology: Learning, Memory, and Cognition, 24, 202-214.

Curiel, J. M., \& Radvansky, G. A. (2002). Mental maps in memory retrieval and comprehension. Memory, 10, 113-126.

Dutke, S. (2003). Anaphor resolution as a function of spatial distance and priming: Exploring the spatial distance effect in situation models. Experimental Psychology, 50, 270-284.

Dutke, S., Ribback, S., \& Wagner, B. (2003). Direct distance, route distance, and implied temporal distance: Effects on the accessibility of situation model components. Scientific Studies of Reading, 7, 357-381.

Dutke, S., \& Rinck, M. (2006). Predictability of locomotion: Effects on updating of spatial situation models during narrative comprehension. Memory \& Cognition, 34, 1193-1205. doi:10.3758/BF03193265

Friedman, A., \& Brown, N. R. (2000). Reasoning about geography. Journal of Experimental Psychology: General, 129, 193-219. doi:10.1037/0096-3445.129.2.193
Glenberg, A. M., Meyer, M., \& Lindem, K. (1987). Mental models contribute to foregrounding during text comprehension. Journal of Memory and Language, 26, 69-83. doi:10.1016/0749596X(87)90063-5

Haenggi, D., Kintsch, W., \& Gernsbacher, M. A. (1995). Spatial situation models and text comprehension. Discourse Processes, 19, 173-200.

Hasher, L., \& Zacks, R. T. (1988). Working memory, comprehension, and aging: A review and a new view. G. H. Bower (Ed.), The psychology of learning and motivation: Advances in research and theory (Vol. 22, pp. 193-225). San Diego, CA: Academic Press. doi:10.1016/S0079-7421(08)60041-9

Kurby, C. A., \& Zacks, J. M. (2008). Segmentation in the perception and memory of events. Trends in Cognitive Sciences, 12, 72-79. doi:10.1016/j.tics.2007.11.004

McNamara, T. P. (1986). Mental representations of spatial relations. Cognitive Psychology, 18, 87-121.

Morrow, D. G., Bower, G. H., \& Greenspan, S. L. (1989). Updating situation models during narrative comprehension. Journal of Memory and Language, 28, 292-312.

Morrow, D. G., Greenspan, S. L., \& Bower, G. H. (1987). Accessibility and situation models in narrative comprehension. Journal of Memory and Language, 26, 165-187. doi:10.1016/0749596X(87)90122-7

Radvansky, G. A. (1998). The organization of information retrieved from situation models. Psychonomic Bulletin \& Review, 5, 283 289. doi:10.3758/BF03212952

Radvansky, G. A. (1999a). The fan effect: A tale of two theories. Journal of Experimental Psychology: General, 128, 198-206.

Radvansky, G. A. (1999b). Memory retrieval and suppression: The inhibition of situation models. Journal of Experimental Psychology: General, 128, 563-579. doi:10.1037/0096-3445.128.4.563

Radvansky, G. A. (2005). Situation models, propositions, and the fan effect. Psychonomic Bulletin \& Review, 12, 478-483. doi:10.3758/BF03193791

Radvansky, G. A. (2009). Spatial directions and situation model organization. Memory \& Cognition, 37, 796-806. doi:10.3758/ MC.37.6.796

Radvansky, G. A. (2012). Across the event horizon. Current Directions in Psychological Science, 21, 269-272. doi:10.1177/ 0963721412451274

Radvansky, G. A., \& Copeland, D. E. (2001). Working memory and situation model updating. Memory \& Cognition, 29, 1073-1080. doi:10.3758/BF03206375

Radvansky, G. A., \& Copeland, D. E. (2006a). Memory retrieval and interference: Working memory issues. Journal of Memory and Language, 55, 33-46.

Radvansky, G. A., \& Copeland, D. E. (2006b). Situation models and retrieval interference: Pictures and words. Memory, 14, 614-623.

Radvansky, G. A., \& Copeland, D. E. (2006c). Walking through doorways causes forgetting: Situation models and experienced space. Memory \& Cognition, 34, 1150-1156. doi:10.3758/ BF03193261

Radvansky, G. A., \& Copeland, D. E. (2010). Reading times and the detection of event shift processing. Journal of Experimental Psychology: Learning, Memory, and Cognition, 36, 210-216. doi: $10.1037 / \mathrm{a} 0017258$

Radvansky, G. A., Krawietz, S. A., \& Tamplin, A. K. (2011). Walking through doorways causes forgetting: Further explorations. Quarterly Journal of Experimental Psychology, 64, 1632-1645. doi:10.1080/ 17470218.2011.571267

Radvansky, G. A., Spieler, D. H., \& Zacks, R. T. (1993). Mental model organization. Journal of Experimental Psychology: Learning, Memory, and Cognition, 19, 95-114.

Radvansky, G. A., \& Tamplin, A. K. (2012). Suppression in retrieval practice, part-set cueing, and negative priming memory: The 
hydrogen model. Quarterly Journal of Experimental Psychology. doi:10.1080/17470218.2012.743572

Radvansky, G. A., Tamplin, A. K., \& Krawietz, S. A. (2010). Walking through doorways causes forgetting: Environmental integration. Psychonomic Bulletin \& Review, 17, 900-904. doi:10.3758/ PBR.17.6.900

Radvansky, G., \& Zacks, J. M. (2011). Event perception. Wiley Interdisciplinary Reviews: Cognitive Science, 2, 608-620. doi:10.1002/wcs. 133

Radvansky, G. A., \& Zacks, R. T. (1991). Mental models and the fan effect. Journal of Experimental Psychology: Learning, Memory, and Cognition, 17, 940-953. doi:10.1037/0278-7393.17.5.940

Rapp, D. N., Klug, J. L., \& Taylor, H. A. (2006). Character movement and the representation of space during narrative comprehension. Memory \& Cognition, 34, 1206-1220. doi:10.3758/BF03193266

Rinck, M., \& Bower, G. H. (1995). Anaphor resolution and the focus of attention in situation models. Journal of Memory and Language, 34, 110-131.

Rinck, M., \& Bower, G. H. (2000). Temporal and spatial distance in situation models. Memory \& Cognition, 28, 1310-1320.

Rinck, M., Bower, G. H., \& Wolf, K. (1998). Distance effects in surface structures and situation models. Scientific Studies of Reading, 2, 221-246.

Rinck, M., \& Denis, M. (2004). The metrics of spatial distance traversed during mental imagery. Journal of Experimental Psychology: Learning, Memory, and Cognition, 30, 1211-1218.

Rinck, M., Hähnel, A., Bower, G. H., \& Glowalla, U. (1997). The metrics of spatial situation models. Journal of Experimental
Psychology: Learning, Memory, and Cognition, 23, 622-637. doi:10.1037/0278-7393.23.3.622

Rinck, M., Williams, P., Bower, G. H., \& Becker, E. S. (1996). Spatial situation models and narrative understanding: Some generalizations and extensions. Discourse Processes, 21, 23-55.

Stevens, A., \& Coupe, P. (1978). Distortions in judged spatial relations. Cognitive Psychology, 10, 422-437.

Stine-Morrow, E. A. L., Morrow, D. G., \& Leno, R. (2002). Aging and the representation of spatial situations in narrative understanding. Journals of Gerontology, 57B, P291-P297.

Swallow, K. M., Zacks, J. M., \& Abrams, R. A. (2009). Event boundaries in perception affect memory encoding and updating. Journal of Experimental Psychology: General, 138, 236-257. doi:10.1037/ a0015631

Thorndyke, P. W. (1981). Distance estimation from cognitive maps. Cognitive Psychology, 13, 526-550.

Van Selst, M., \& Jolicœur, P. (1994). A solution to the effect of sample size on outlier estimation. Quarterly Journal of Experimental Psychology, 47A, 631-650. doi:10.1080/14640749408401131

Wilson, S. G., Rinck, M., McNamara, T. P., Bower, G. H., \& Morrow, D. G. (1993). Mental models and narrative comprehension: Some qualifications. Journal of Memory and Language, $32,141-154$

Zwaan, R. A., \& Radvansky, G. A. (1998). Situation models in language comprehension and memory. Psychological Bulletin, 123, 162-185. doi:10.1037/0033-2909.123.2.162

Zwaan, R. A., Radvansky, G. A., Hilliard, A. E., \& Curiel, J. M. (1998). Constructing multidimensional situation models during reading. Scientific Studies of Reading, 2, 199-220. 